\title{
Clinical intervals and diagnostic characteristics in a cohort of prostate cancer patients in Spain: a multicentre observational study
}

Xavier Bonfill ${ }^{1,2,3,4}$, María José Martinez-Zapata ${ }^{1,2,3^{*}}$, Robin WM Vernooij², María José Sánchez ${ }^{1,5}$, María Morales Suárez-Varela ${ }^{1,6}$, Javier de la Cruz ${ }^{1,7}$, José Ignacio Emparanza ${ }^{1,8}$, Montserrat Ferrer ${ }^{1,9}$, José Ignacio Pijoán ${ }^{1,10}$, Juan M. Ramos-Goñi ${ }^{11}$, Joan Palou ${ }^{3,12}$, Stefanie Schmidt ${ }^{13}$, Víctor Abraira ${ }^{1,14}$, Javier Zamora ${ }^{1,14}$ and on behalf of the EMPARO-CU study group

\begin{abstract}
Background: Little is known about the healthcare process for patients with prostate cancer, mainly because hospital-based data are not routinely published. The main objective of this study was to determine the clinical characteristics of prostate cancer patients, the, diagnostic process and the factors that might influence intervals from consultation to diagnosis and from diagnosis to treatment.

Methods: We conducted a multicentre, cohort study in seven hospitals in Spain. Patients' characteristics and diagnostic and therapeutic variables were obtained from hospital records and patients' structured interviews from October 2010 to September 2011. We used a multilevel logistic regression model to examine the association between patient care intervals and various variables influencing these intervals (age, BMl, educational level, ECOG, first specialist consultation, tumour stage, PSA, Gleason score, and presence of symptoms) and calculated the odds ratio (OR) and the interquartile range (IQR). To estimate the random inter-hospital variability, we used the median odds ratio (MOR).

Results: 470 patients with prostate cancer were included. Mean age was 67.8 (SD: 7.6) years and $75.4 \%$ were physically active. Tumour size was classified as T1 in $41.0 \%$ and as T2 in $40 \%$ of patients, their median Gleason score was 6.0 (IQR:1.0), and $36.1 \%$ had low risk cancer according to the D'Amico classification. The median interval between first consultation and diagnosis was 89 days (IQR:123.5) with no statistically significant variability between centres. Presence of symptoms was associated with a significantly longer interval between first consultation and diagnosis than no symptoms (OR:1.93, 95\% Cl 1.29-2.89). The median time between diagnosis and first treatment (therapeutic interval) was 75.0 days (IQR:78.0) and significant variability between centres was found (MOR:2.16, 95\%Cl 1.45-4.87). This interval was shorter in patients with a high PSA value $(p=0.012)$ and a high Gleason score $(p=0.026)$.
\end{abstract}

Conclusions: Most incident prostate cancer patients in Spain are diagnosed at an early stage of an adenocarcinoma. The period to complete the diagnostic process is approximately three months whereas the therapeutic intervals vary among centres and are shorter for patients with a worse prognosis. The presence of prostatic symptoms, PSA level, and Gleason score influence all the clinical intervals differently.

Keywords: Prostatic neoplasms, Male urogenital diseases, Multicentre study, Cohort study, Prospective study

\footnotetext{
* Correspondence: mmartinezz@santpau.cat

${ }^{1}$ CIBER de Epidemiología y Salud Pública (CIBERESP), Madrid, Spain

${ }^{2}$ Institute of Biomedical Research (IIB Sant Pau), Iberoamerican Cochrane

Centre, Barcelona, Spain

Full list of author information is available at the end of the article
}

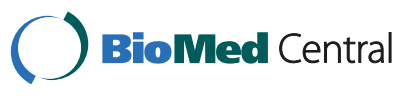

(c) 2015 Bonfill et al. This is an Open Access article distributed under the terms of the Creative Commons Attribution License (http://creativecommons.org/licenses/by/4.0), which permits unrestricted use, distribution, and reproduction in any medium, provided the original work is properly credited. The Creative Commons Public Domain Dedication waiver (http:// creativecommons.org/publicdomain/zero/1.0/) applies to the data made available in this article, unless otherwise stated. 


\section{Background}

Prostate cancer is the most frequently diagnosed cancer among Spanish men. With an incidence of 65.2 per 100 000 persons per year (27 853 new cases yearly $21.7 \%$ of the total cancer in men), it is overall the second most frequent cancer in Spain [1]. Worldwide, it is the second most frequently diagnosed cancer among men (1 111 689 new cases, $15.0 \%$ of all cancers in men) and overall the fourth most common cancer [1]. The incidence of prostate cancer has increased over the last decades, partly due to the more frequent use of diagnostic tools such as prostate-specific antigen (PSA) testing and needle biopsies in asymptomatic men [2-4]. The impact on mortality is high. Mortality rates in Spain showed a slight increase between 1980 and 1998 but have since decreased [5, 6]. In 2012, the estimated mortality associated with prostate cancer was 5481 in Spain and 307 471 worldwide, making it the third leading cause of death due to cancer for men in Spain (8.6\% of the total) and the sixth leading cause worldwide $(6.6 \%$ of the total) [1]. Furthermore, prostate cancer reduces the quality of life of patients $[7,8]$.

The economic burden of prostate cancer is one of the largest among malignant tumours due to the high incidence of the disease and increasing survival rates [9]. It is estimated to cost 11.85 billion USD annually in the USA [9]. Total costs for diagnosing, treating, and monitoring patients with prostate cancer for five years have been estimated to be approximately $£ 7294.2$ per patient and $£ 92.74$ million overall in the United Kingdom [10].

The Spanish Health System is funded by taxes. It offers universal coverage and is managed regionally within each of the 17 autonomous communities. Healthcare is divided into two broad areas, primary care and hospital care. Prostate cancer is generally detected in primary care centres, where patients might undergo some diagnostic tests. For confirmatory tests, however, such as a prostate biopsy, the patient is referred to a hospital for specialised healthcare. Direct access to specialised healthcare may also occur through the hospital emergency services, but this is less frequent.

Several international initiatives have been launched to obtain detailed and reliable information regarding the healthcare process for prostate cancer patients. This information includes the time intervals between first consultation to diagnosis, and first treatment. Such projects include The European Cancer Registry-based Study of Survival and Care of Cancer Patients (EUROCARE) [11], the Patient Outcome Research Teams (PORTS) [12], and the Cancer of the Prostate Strategic Urologic Research Endeavour (CAPSURE) [13]. Information can also be obtained from databases containing regional and national incidence and mortality statistics, from hospital minimum data sets, and from hospital-based cancer registries that allow a description and generic comparison of hospital healthcare [14, 15]. These sources of information, however, do not include the type of data needed to identify the diagnostic processes, therapeutic approaches, and prognostic factors in prostate cancer. Recently, one study regarding prostate cancer has been conducted in Spain, with the objective to estimate prostate cancer incidence and profile the newly-diagnosed cases using a nationwide hospital-based registry [16, 17]. However, this study fails to examine the diagnosis and therapeutic processes and possible factors influencing these time intervals. The objective of the EMPARO-CU study is to examine the clinical care process and health outcomes of patients with urologic tumours during the first year from the histopathological prostate cancer confirmation. In this paper we describe the patients' baseline characteristics at hospital entry and the time intervals between the first consultation and diagnosis, and between diagnosis and start of treatment and possible factors influencing these intervals.

\section{Methods}

The EMPARO-CU study is a multicentre, cohort study of bladder and prostate cancer, conducted in seven tertiary hospitals in Spain: Fundació Puigvert-Hospital de la Santa Creu i Sant Pau (coordinating centre) and Hospital del Mar in Barcelona, Hospital Universitario 12 de Octubre and Hospital Universitario Ramón y Cajal in Madrid, Hospital Universitario Donostia in Donostia-San Sebastián, Hospital General Universitario de Valencia in Valencia, and Hospital Universitario Virgen de las Nieves in Granada (list of participants in Appendix). The protocol was approved by the research ethics committees at each participating centre (Table 1). Patients were enrolled from October 2010 to September 2011. Consecutive patients were selected from the urologic and oncology departments at each centre. Inclusion criteria were: 1 ) diagnosis of prostate cancer during the study period, independently of the tumour stage; 2) diagnosis and treatment at one of the participating hospitals; and 3) agreement to participate and signed informed consent.

Table 1 List of ethic committees that approval the study Hospital de la Santa Creu i Sant Pau (Barcelona)

Fundación Puigvert (Barcelona)

Hospital 12 de Octubre (Madrid)

Hospital Ramón y Cajal (Madrid)

Autonómico del País Vasco

Hospital Donosti (San Sebastián)

Hospital General Universitario de Valencia

Hospital Nuestra Señora del Mar (Barcelona)

Hospital Virgen de las Nieves (Granada) 
The EMPARO-CU study focuses on the clinical care process and health outcomes of patients with urologic tumours. In this paper we describe the patients' baseline characteristics at hospital entry and the intervals between the first consultation and diagnosis, and between diagnosis and start of treatment. Information regarding patient status before the diagnosis (such as symptoms at first visit) was collected retrospectively. Study data were collected from the medical records and from structured interviews with individual patients. Variables of interest were: socio-demographic data, body mass index (BMI), Charlson index, ECOG WHO score, first specialist consulted, diagnostic tests performed to establish a diagnosis of prostate cancer, pathological results of prostate biopsy [18], PSA values, total Gleason scores, clinical stages, time from first symptom to first consultation, and time from first consultation to primary diagnosis and first treatment (Fig. 1). Time from first symptom to first consultation was defined as the date on which the patients experienced the first symptoms related to prostate cancer. The date of first consultation was considered the date on which the patient first consulted a healthcare professional for the symptoms that led to prostate cancer screening. For asymptomatic patients, the first consultation was considered the date on which the physician performed prostate screening. We considered the first histological confirmation as the confirmatory diagnosis of the disease. The reference date to calculate intervals was the date of biopsy that confirmed the histological diagnosis of prostate cancer. The time interval between the first consultation and biopsy was considered the diagnostic interval. The time between the biopsy and first treatment was considered the therapeutic interval.

Categorical variables are described using relative frequency, and continuous variables are described using mean and standard deviation (SD) or median and interquartile range (IQR) for skewed distribution variables. The frequency of missing values is reported for each variable.

The association between time variables and potential predictors was assessed using multilevel (patients at first level and hospitals at second level) logistic regression models. The variables included as potential predictors in both models were age, BMI, education level, ECOG WHO score, specialist at first consultation, primary tumour clinical stage, PSA value, Gleason score, and presence of prostate cancer symptoms. Continuous time variables were transformed into dichotomous variables. In agreement with previous studies, cut-offs chosen were an interval of 100 days between first consultation and diagnosis and 30 days between diagnosis and treatment $[19,20]$. These intervals were based on recommendations about optimal diagnostic and therapeutic intervals [21, 22]. We first fitted an empty model that considered only the random effect of the hospital on the variability

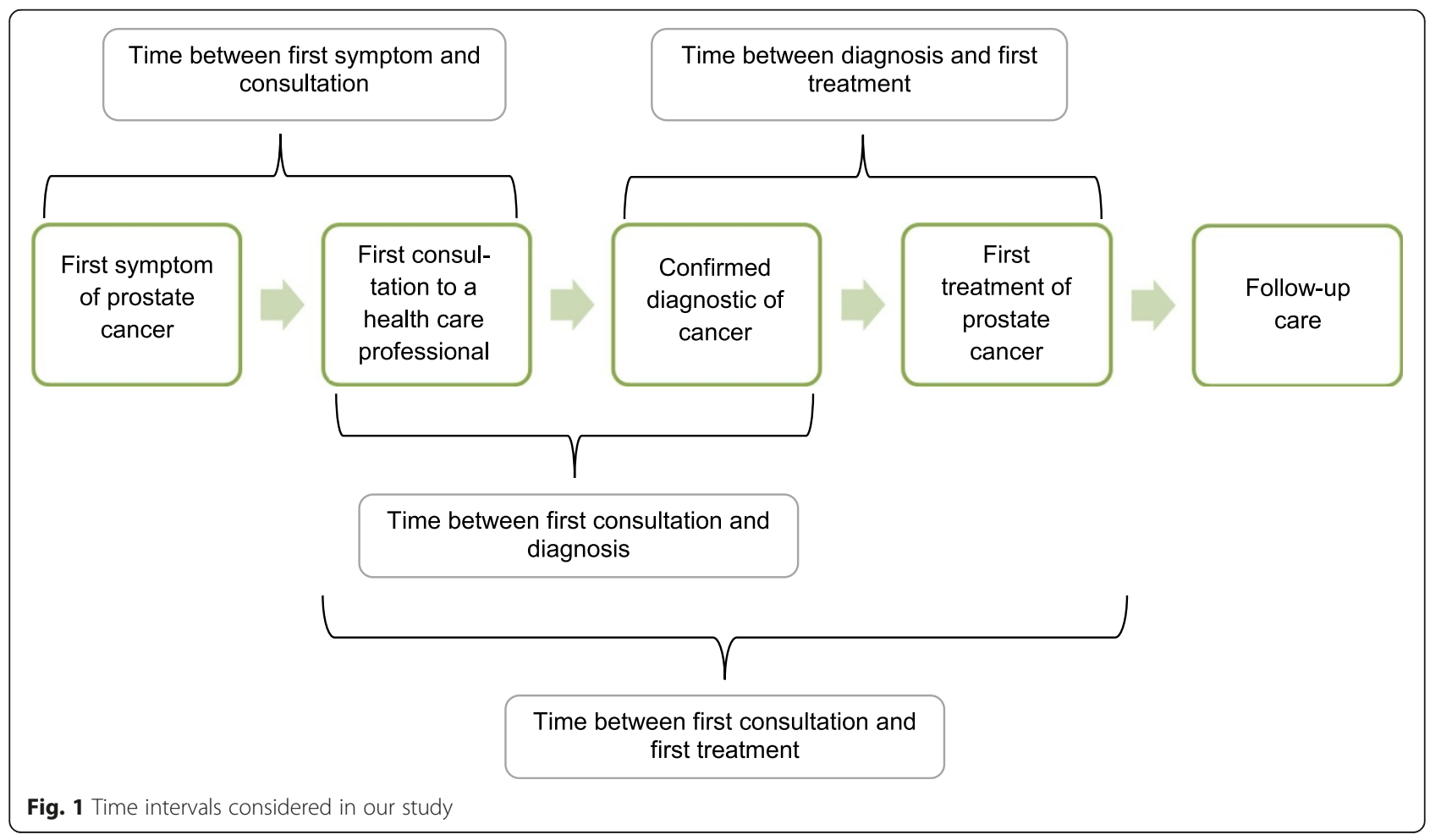


Table 2 Characteristics of prostate cancer patients

\begin{tabular}{|c|c|}
\hline Variables & $N=470$ \\
\hline & n (\%) \\
\hline Mean age \pm SD & $67.8 \pm 7.6$ \\
\hline Missing (\%): & 2.3 \\
\hline Mean $\mathrm{BMI} \pm \mathrm{SD}$ & $28.1 \pm 4.5$ \\
\hline Missing (\%) & 3.8 \\
\hline \multicolumn{2}{|l|}{ Working status } \\
\hline Active & $83(17.7)$ \\
\hline Sick leave & $15(3.1)$ \\
\hline Retired & $347(73.8)$ \\
\hline Unemployed & $17(3.6)$ \\
\hline Other & $4(0.9)$ \\
\hline Missing & $4(0.9)$ \\
\hline \multicolumn{2}{|l|}{ Education (\%) } \\
\hline No education & $47(10.2)$ \\
\hline Incomplete primary education & $80(17.2)$ \\
\hline Primary education & $109(23.5)$ \\
\hline Graduate school & $99(21.3)$ \\
\hline Upper secondary studies & $62(13.4)$ \\
\hline University & $67(14.5)$ \\
\hline Missing & $6(1.2)$ \\
\hline \multicolumn{2}{|l|}{ Setting first consultation (\%) } \\
\hline Primary care & $355(75.5)$ \\
\hline Hospital & $86(18.5)$ \\
\hline Other & $23(5.0)$ \\
\hline Missing & $6(1.2)$ \\
\hline \multicolumn{2}{|l|}{ Symptoms (\%) } \\
\hline No symptoms or discomfort & $251(53.4)$ \\
\hline One or more symptoms & $170(36.2)$ \\
\hline Missing & $49(10.4)$ \\
\hline \multicolumn{2}{|c|}{$\begin{array}{l}\text { Start of first symptoms including patients with } \\
\text { discomfort (\%) }\end{array}$} \\
\hline Since one month & $55(11.7)$ \\
\hline Between one month and one year & $242(51.4)$ \\
\hline Later than a year & $56(12.0)$ \\
\hline No symptoms & $68(14.5)$ \\
\hline Missing & $49(10.4)$ \\
\hline \multicolumn{2}{|l|}{ ECOG WHO (\%) } \\
\hline Fully active: & $356(75.7)$ \\
\hline Restricted or worse: & $105(22.3)$ \\
\hline Missing: & $9(2.0)$ \\
\hline \multicolumn{2}{|l|}{ Charlson index (\%) } \\
\hline 1: & $358(75.8)$ \\
\hline 2: & $68(14.4)$ \\
\hline 3: & $26(5.5)$ \\
\hline
\end{tabular}

Table 2 Characteristics of prostate cancer patients (Continued)

\begin{tabular}{|c|c|}
\hline 4: & $13(2.8)$ \\
\hline$\geq 5:$ & $5(1.5)$ \\
\hline Median PSA $(\mathrm{ng} / \mathrm{mL}) \pm \mathrm{IQR}$ & $7.6 \pm 7.8$ \\
\hline Missing (\%): & 3.4 \\
\hline \multicolumn{2}{|l|}{ Total Gleason (\%) } \\
\hline 2-6: & $262(55.6)$ \\
\hline 7: & $127(26.9)$ \\
\hline$>7:$ & $74(15.6)$ \\
\hline Missing: & $7(1.9)$ \\
\hline Median total Gleason \pm IQR & $6.0 \pm 1.0$ \\
\hline \multicolumn{2}{|l|}{ Primary tumour clinical stage (T) (\%) } \\
\hline Tx: & $1(0.2)$ \\
\hline T1a-c & $194(41.0)$ \\
\hline T2a-c: & $189(40.1)$ \\
\hline T3a-b : & $74(15.7)$ \\
\hline T4: & $8(1.7)$ \\
\hline Missing: & $4(1.3)$ \\
\hline \multicolumn{2}{|l|}{ Regional lymph nodes clinical stage (N) (\%) } \\
\hline Nx-NO: & $460(97.7)$ \\
\hline N1: & $10(2.3)$ \\
\hline Missing: & $0(0.0)$ \\
\hline \multicolumn{2}{|l|}{ Distance metastasis clinical stage (M) (\%) } \\
\hline Mx-MO: & $460(97.9)$ \\
\hline M1a-c: & $10(2.1)$ \\
\hline Missing: & $0(0.0)$ \\
\hline \multicolumn{2}{|l|}{ D’Amico Classification (\%) } \\
\hline Low risk: & $170(36.1)$ \\
\hline Medium risk: & $115(24.5)$ \\
\hline High risk: & $185(39.4)$ \\
\hline Missing: & $0(0.0)$ \\
\hline $\begin{array}{l}\text { Median time between first consultation and } \\
\text { diagnosis in days } \pm \text { IQR }\end{array}$ & $89.0 \pm 123.5$ \\
\hline Missing (\%): & 4.0 \\
\hline $\begin{array}{l}\text { Median time between diagnosis and first } \\
\text { treatment in days } \pm \text { IQR }\end{array}$ & $75.0 \pm 78.0$ \\
\hline Missing (\%): & 3.2 \\
\hline $\begin{array}{l}\text { Median time between first consultation and } \\
\text { first treatment in days } \pm I Q R\end{array}$ & $176.0 \pm 151.0$ \\
\hline Missing (\%): & 6.6 \\
\hline
\end{tabular}

of the two outcomes investigated. We then fitted univariate models with each potential predictor. The final model was fitted through a backward selection procedure based on Wald tests results. Both the empty model and the final multilevel models were estimated by maximum likelihood based on Gaussian quadrature points [23]. 
To estimate the random inter-hospital variability, we used the intra-cluster correlation coefficient (ICC) and the median odds ratio (MOR). The ICC indicates the fraction of the total outcome variability that is attributable to the area level (in our case, hospital level) and provides a measure of the within-hospital homogeneity. A lower ICC indicates a lower likelihood of patients' sharing hospital experiences. However, because the ICC can be difficult to interpret because of binary outcomes, the partition of variance between different levels does not have the intuitive interpretation of the linear model. We therefore also calculated the MOR, defined as the median value of the odds ratio between the hospital at highest risk (longest time interval) and the hospital at lowest risk when randomly picking out two hospitals. The MOR can be conceptualised as the increased risk (in median) that a patient would have if moved to a hospital with a higher risk [24]. The measure of fixed effect was the odds ratio (OR) with $95 \%$ confidence intervals. A $p$-value lower than 0.05 was considered statistically significant for all statistical analyses. Data analyses were performed using SPSS statistical software, version 20.0 (SPSS INC., Chicago, IL, USA) and Stata v12 (StataCorp. 2011. Stata Statistical Software: Release 12. College Station, TX: StataCorp LP).

\section{Results}

Of the 502 patients recruited, 32 were excluded because they did not meet the inclusion criteria. The study group was therefore composed of 470 patients. Mean age was 67.8 years (SD:7.6), 337 (71.9\%) had completed at least primary studies, and 347 (73.8 \%) were retired (Table 2). The mean BMI was 28.1 (SD:4.5) and 354 (75.4\%) had no physical limitations according to the ECOG WHO performance status. The Charlson comorbidity index was between one and three for 451 participants (96.2\%). Prostate screening was performed in primary care settings for 355 of the 470 patients (75.5\%), and in hospital settings for 86 of these participants (18.3\%). In $53.4 \%$ of patients, the disease was identified during a routine visit or during consultation for another cause because no symptoms or only discomfort caused by prostate cancer had been noted. The median PSA value for the patients without symptoms was 7.2 (IQR: 6.9). From the total group, $36.2 \%$ were symptomatic; $48.1 \%$ of these patients had lower urinary tract symptoms such as increased frequency of urination (16.3\%), and $7.6 \%$ had symptoms related to the tumour. The time from the first symptom to first consultation was between one month and one year for $50.8 \%$ of participants. The clinical stage of the primary tumour was T1a-c in $41.0 \%$ cases and T2 a-c in $40 \%$ cases; $2.3 \%$ had regional lymph nodes (N1) and $2.3 \%$ had distant metastases (M1). The median PSA value was 7.6 (IQR: 7.8) $\mathrm{ng} / \mathrm{mL}$ and the total Gleason score was between two and six for $55.6 \%$ of participants. According to the D'Amico classification, $36.1 \%$ of patients had low-risk cancer and $39.4 \%$ had high-risk cancer (Table 2).

All patients had a prostate biopsy and $82.9 \%$ underwent a prostate ultrasound study. A renal ultrasonography was performed in $23.8 \%$ of patients and a bladder ultrasonography in $22.5 \%$ (Table 3 ). Table 4 shows the patients' characteristics for each participating hospital. The median diagnostic interval was 89.0 days (IQR: 123.5). No statistically significant differences were found between hospitals for this interval (MOR: 1.00). Patients with one or more symptoms had an OR of 1.93 (95\% CI 1.29-2.89, $P=0.001$ ) of having an interval between first consultation and diagnosis of more than 100 days (Table 5). No significant differences were found for groups of patients differing in age, BMI, education level, ECOG WHO score, the specialist at first consultation, primary tumour stage, PSA, or total Gleason scores.

The median therapeutic interval was 75 days (IQR: 78.0) (Table 5). No statistically significant association was found between groups for this interval regarding age, BMI, education level, specialist at first consultation, or primary tumour stage (Table 6). A higher PSA value and a higher Gleason score shortened the interval between diagnosis and treatment. Patients with a PSA value higher than 10 or a total Gleason score higher than 7 had an OR of 0.5 (95\% CI 0.29-0.86, $P=0.012$ ) and 0.53 (95\% CI $0.30-0.93, P=0.026$ ), respectively, to

Table 3 Diagnostic variables of prostate cancer patients

\begin{tabular}{ll}
\hline Diagnostic test & $\mathrm{N}=470$ \\
$\mathrm{n}(\%)$
\end{tabular}


Table 4 Characteristics of prostate cancer patients by hospitals

\begin{tabular}{|c|c|c|c|c|c|c|c|}
\hline Centres & $\mathrm{A}(n=48)$ & $\mathrm{B}(n=91)$ & $C(n=37)$ & $\mathrm{D}(n=78)$ & $\mathrm{E}(n=112)$ & $\mathrm{F}(n=33)$ & $\mathrm{G}(n=75)$ \\
\hline Mean age $\pm S D$ & $72.6 \pm 6.1$ & $66.9 \pm 7.7$ & $67.3 \pm 5.6$ & $67.8 \pm 6.7$ & $67.1 \pm 7.8$ & $66.2 \pm 6.9$ & $67.6 \pm 9.2$ \\
\hline Missing (\%): & 0.0 & 2.2 & 0.0 & 5.1 & 0.0 & 6.1 & 4.1 \\
\hline Mean $\mathrm{BMI} \pm \mathrm{SD}$ & $28.2 \pm 5.7$ & $27.3 \pm 3.5$ & $27.6 \pm 3.8$ & $28.8 \pm 6.4$ & $28.9 \pm 4.1$ & $27.6 \pm 4.3$ & $27.2 \pm 2.9$ \\
\hline Missing (\%): & 0.0 & 4.4 & 0.0 & 3.8 & 0.9 & 21.2 & 4.1 \\
\hline Median PSA $(\mathrm{ng} / \mathrm{mL}) \pm I Q R$ & $10.4 \pm 15.5$ & $5.7 \pm 3.4$ & $6.7 \pm 3.7$ & $7.8 \pm 7.7$ & $8.6 \pm 12.2$ & $7.0 \pm 3.8$ & $8.7 \pm 8.2$ \\
\hline Missing (\%): & 2.1 & 8.8 & 0.0 & 6.4 & 0.0 & 3.0 & 1.4 \\
\hline \multicolumn{8}{|l|}{ ECOG WHO (\%) } \\
\hline Fully active: & 72.9 & 84.6 & 91.9 & 71.8 & 69.6 & 63.6 & 75.3 \\
\hline Restricted or worse: & 25.0 & 15.4 & 8.1 & 23.1 & 30.4 & 33.3 & 17.8 \\
\hline Missing: & 2.1 & 0.0 & 0.0 & 5.1 & 0.0 & 3.0 & 6.8 \\
\hline \multicolumn{8}{|l|}{ Primary tumour clinical stage (T) (\%) } \\
\hline Tx: & 0.0 & 0.0 & 0.0 & 1.3 & 1.3 & 0.0 & 0.0 \\
\hline T1a-c & 45.8 & 45.1 & 16.2 & 37.2 & 37.2 & 43.8 & 41.1 \\
\hline T2a-c: & 43.8 & 39.6 & 56.8 & 37.2 & 37.2 & 33.0 & 19.3 \\
\hline T3a-b : & 6.3 & 14.3 & 21.6 & 20.5 & 20.5 & 22.3 & 6.8 \\
\hline T4: & 4.1 & 1.0 & 0.0 & 3.8 & 3.8 & 0.9 & 1.4 \\
\hline Missing: & 0.0 & 0.0 & 5.4 & 0.0 & 0.0 & 0.0 & 1.4 \\
\hline \multicolumn{8}{|l|}{ Regional lymph nodes clinical stage (N) (\%) } \\
\hline Nx-NO: & 100.0 & 98.9 & 100.0 & 92.3 & 100.0 & 100.0 & 97.3 \\
\hline N1: & 0.0 & 1.1 & 0.0 & 7.7 & 0.0 & 0.0 & 2.7 \\
\hline Missing: & 0.0 & 0.0 & 0.0 & 0.0 & 0.0 & 0.0 & 0.0 \\
\hline \multicolumn{8}{|l|}{ Distance metastasis clinical stage (M) (\%) } \\
\hline Mx-Mo: & 95.8 & 100.0 & 100.0 & 93.6 & 98.2 & 100.0 & 98.6 \\
\hline M1a-c: & 4.2 & 0.0 & 0.0 & 6.4 & 1.8 & 0.0 & 1.4 \\
\hline Missing: & 0.0 & 0.0 & 0.0 & 0.0 & 0.0 & 0.0 & 0.0 \\
\hline \multicolumn{8}{|l|}{ D’Amico Classification (\%) } \\
\hline Low risk: & 14.6 & 50.5 & 54.1 & 32.1 & 39.3 & 36.4 & 21.9 \\
\hline Medium risk: & 27.1 & 12.1 & 29.7 & 17.9 & 23.2 & 45.5 & 34.2 \\
\hline High risk: & 58.3 & 37.4 & 16.2 & 50.0 & 37.5 & 18.1 & 42.5 \\
\hline Missing: & 0.0 & 0.0 & 0.0 & 0.0 & 0.0 & 0.0 & 1.4 \\
\hline \multicolumn{8}{|l|}{ Total Gleason (\%) } \\
\hline 2-6: & 16.7 & 73.6 & 64.9 & 68.0 & 66.9 & 36.4 & 31.5 \\
\hline 7: & 29.2 & 19.8 & 29.7 & 17.9 & 18.8 & 51.5 & 43.8 \\
\hline$>7:$ & 54.1 & 6.6 & 2.7 & 10.2 & 14.3 & 9.1 & 19.2 \\
\hline Missing: & 0.0 & 0.0 & 2.7 & 3.9 & 0.0 & 3.0 & 5.5 \\
\hline Median total Gleason \pm IQR & $8.0 \pm 1.0$ & $6.0 \pm 1.0$ & $6.0 \pm 1.0$ & $6.0 \pm 2.0$ & $6.0 \pm 1.0$ & $7.0 \pm 1.0$ & $7.0 \pm 1.0$ \\
\hline $\begin{array}{l}\text { Median time between first consultation } \\
\text { and diagnosis in days } \pm \text { IQR }\end{array}$ & $99.5 \pm 139.0$ & $79.0 \pm 211.0$ & $110.0 \pm 117.0$ & $78.5 \pm 107.3$ & $92.0 \pm 99.0$ & $133.0 \pm 195.0$ & $76.5 \pm 100.0$ \\
\hline Missing (\%): & 0.0 & 3.3 & 5.4 & 7.7 & 4.5 & 6.1 & 1.4 \\
\hline $\begin{array}{l}\text { Median time between diagnosis and } \\
\text { first treatment in days } \pm \mathrm{IQR}\end{array}$ & $30.5 \pm 127.5$ & $78.0 \pm 62.0$ & $83.0 \pm 72.0$ & $104.0 \pm 70.5$ & $55.0 \pm 66.5$ & $73.5 \pm 84.5$ & $70.0 \pm 95.0$ \\
\hline Missing (\%): & 0.0 & 4.4 & 0.0 & 1.3 & 6.3 & 3.0 & 2.7 \\
\hline $\begin{array}{l}\text { Median time between first consultation } \\
\text { and first treatment in days } \pm \mathrm{IQR}\end{array}$ & $164.0 \pm 232.0$ & $154.0 \pm 207.0$ & $212.0 \pm 165.0$ & $200.0 \pm 130.0$ & $166.0 \pm 112.8$ & $203.0 \pm 149.8$ & $165.0 \pm 128.5$ \\
\hline Missing (\%): & 0.0 & 6.6 & 5.4 & 9.0 & 8.9 & 9.1 & 4.1 \\
\hline
\end{tabular}


Table 5 Time interval between first consultancy and diagnosis and potential determinant (Univariate regression)

\begin{tabular}{|c|c|c|c|c|c|}
\hline & & & ICC/MOR & $95 \% \mathrm{Cl}$ MOR & $P$-value \\
\hline Hospital random effect & & & 0.00 & & \\
\hline \multirow[t]{2}{*}{ Empty model } & & & 1.00 & $1.00-1.00$ & 1.000 \\
\hline & Median (days) & IQR (days) & OR $>100$ days & $95 \% \mathrm{Cl} O \mathrm{R}$ & \\
\hline \multicolumn{6}{|l|}{ Age } \\
\hline$<65$ years & 84.0 & 182.0 & 1 & & \\
\hline$\geq 65$ years & 91.0 & 105.5 & 0.96 & $0.64-1.43$ & 0.830 \\
\hline \multicolumn{6}{|l|}{ BMl } \\
\hline$<25$ & 102.0 & 163.0 & 1 & & \\
\hline$\geq 25$ & 86.0 & 110.3 & 0.64 & $0.40-1.01$ & 0.057 \\
\hline \multicolumn{6}{|l|}{ Education level } \\
\hline Primary education or lower & 90.5 & 108.5 & 1 & & \\
\hline Graduate school or higher & 86.0 & 139.0 & 1.00 & $0.69-1.45$ & 0.992 \\
\hline \multicolumn{6}{|l|}{ ECOG WHO Score } \\
\hline Fully active & 87.5 & 126.8 & 1 & & \\
\hline Restrictive or worse & 95.0 & 129.0 & 1.18 & $0.76-1.83$ & 0.474 \\
\hline \multicolumn{6}{|l|}{ Specialist first consultation } \\
\hline Primary care & 91.0 & 139.5 & 1 & & \\
\hline Hospital or specialist & 79.0 & 106.0 & 0.78 & $0.50-1.22$ & 0.277 \\
\hline \multicolumn{6}{|l|}{ Primary tumour clinical stage $(T)$} \\
\hline T1a-T1c & 95.5 & 141.3 & 1 & & \\
\hline T2a-T4 & 85.0 & 108.5 & 0.73 & $0.50-1.07$ & 0.110 \\
\hline \multicolumn{6}{|l|}{ PSA value } \\
\hline$<10$ & 95.0 & 135.5 & 1 & & \\
\hline$\geq 10$ & 84.0 & 108.5 & 0.81 & $0.54-1.21$ & 0.310 \\
\hline \multicolumn{6}{|l|}{ Total Gleason score } \\
\hline$<7$ & 87.0 & 140.0 & 1 & & \\
\hline$\geq 7$ & 91.0 & 111.5 & 0.87 & $0.60-1.27$ & 0.464 \\
\hline \multicolumn{6}{|l|}{ Symptoms } \\
\hline No symptoms or discomfort & 83.0 & 110.0 & 1 & & \\
\hline One or more symptoms & 110.0 & 174.0 & 1.93 & $1.29-2.89$ & 0.001 \\
\hline
\end{tabular}

have an interval between diagnosis and treatment of more than 30 days. The MOR for the random effect of the hospital where the patient received care was 2.16 (95\% CI 1.45-4.87, $P=0.000$ ).

\section{Discussion}

This multicentre cohort study aimed to describe the healthcare process in patients with prostate cancer in Spain. We focused on the characteristics of patients and tumours and we evaluated diagnosis and treatment delays in healthcare.

Our study included 470 patients diagnosed with prostate adenocarcinoma in a hospital care setting. Prostate biopsy and ultrasound were the most frequently performed diagnostic tests. The mean age of our population, the proportion of asymptomatic low risk patients and the median Gleason grade were similar to those reported in previous studies in Spain and in other countries $[16,19,25-29]$. The percentage of localised tumours in our population (81\%) was similar to that in an earlier study $(89.8 \%)$ in Spain conducted by Cozar et al. but considerably higher than that in the European study of Gatta et al. These discordant findings might be explained by differences between countries and years regarding accessibility to health services and physicians' attitudes towards screening tests [30].

In our study, clinical symptoms were present in $36.2 \%$ of all patients, the most common symptom being disorders of the lower urinary tract $(48.1 \%)$ and symptoms 
Table 6 Time interval between diagnosis and first treatment and potential determinants

\begin{tabular}{|c|c|c|c|c|c|c|c|c|c|}
\hline \multirow{4}{*}{$\begin{array}{l}\text { Characteristic } \\
\text { Hospital random effect }\end{array}$} & \multicolumn{5}{|c|}{ Univariate regression } & \multicolumn{4}{|c|}{ Multivariate regression } \\
\hline & \multirow[b]{3}{*}{ Median (days) } & \multirow[b]{3}{*}{ IQR (days) } & \multirow{3}{*}{$\begin{array}{l}\text { Empty model } \\
\text { ICC/MOR } \\
0.18 / 2.22 \\
\text { OR }>30 \text { days }\end{array}$} & \multirow{3}{*}{$\begin{array}{l}95 \% \mathrm{Cl} \\
\mathrm{MOR} \\
1.52-4.58 \\
95 \% \mathrm{Cl} \text { OR }\end{array}$} & \multirow{3}{*}{$\begin{array}{l}P \text {-value } \\
0.000\end{array}$} & \multirow[b]{3}{*}{$\mathrm{OR}>30$ days } & \multirow{3}{*}{$\begin{array}{l}\text { Flnal model } \\
\text { ICC/MOR } \\
0.17 / 2.16 \\
95 \% \mathrm{Cl} \text { OR }\end{array}$} & \multirow{3}{*}{$\begin{array}{l}95 \% \mathrm{Cl} \\
\mathrm{MOR} \\
1.45-4.87\end{array}$} & \multirow{3}{*}{$\begin{array}{l}P \text {-value } \\
0.000\end{array}$} \\
\hline & & & & & & & & & \\
\hline & & & & & & & & & \\
\hline \multicolumn{10}{|l|}{ Age } \\
\hline$<65$ years & 78.0 & 74.0 & 1 & & & & & & \\
\hline$\geq 65$ years & 71.0 & 79.0 & 0.70 & $0.40-1.21$ & 0.198 & & & & \\
\hline \multicolumn{10}{|l|}{ BMI } \\
\hline$<25$ & 75.0 & 76.0 & 1 & & & & & & \\
\hline$\geq 25$ & 72.0 & 81.0 & 1.06 & $0.60-1.90$ & 0.831 & & & & \\
\hline \multicolumn{10}{|l|}{ Education level } \\
\hline Primary education or lower & 76.0 & 77.8 & 1 & & & & & & \\
\hline Graduate school or higher & 70.0 & 77.5 & 1.25 & $0.76-2.06$ & 0.386 & & & & \\
\hline \multicolumn{10}{|l|}{ ECOG WHO Score } \\
\hline Fully active & 77.0 & 74.8 & 1 & & & & & & \\
\hline Restrictive or worse & 54.0 & 77.0 & 0.55 & $0.32-0.95$ & 0.033 & & & & \\
\hline \multicolumn{10}{|l|}{ Specialist first consultation } \\
\hline Primary care & 76.0 & 77.0 & 1 & & & & & & \\
\hline Hospital or specialist & 74.0 & 79.5 & 0.86 & $0.48-1.56$ & 0.621 & & & & \\
\hline \multicolumn{10}{|l|}{ Primary tumour clinical stage $(T)$} \\
\hline T1a-T1c & 84.0 & 77.0 & 1 & & & & & & \\
\hline T2a-T4 & 69.0 & 79.0 & 0.59 & $0.35-0.98$ & 0.040 & & & & \\
\hline \multicolumn{10}{|l|}{ PSA value } \\
\hline$<10$ & 86.0 & 75.0 & 1 & & & & & & \\
\hline$\geq 10$ & 50.0 & 81.0 & 0.41 & $0.24-0.68$ & 0.001 & 0.50 & $0.29-0.86$ & & 0.012 \\
\hline \multicolumn{10}{|l|}{ Gleason score } \\
\hline$<7$ & 85.5 & 68.8 & 1 & & & & & & \\
\hline$\geq 7$ & 55.0 & 88.0 & 0.42 & $0.25-0.71$ & 0.001 & 0.53 & $0.30-0.93$ & & 0.026 \\
\hline \multicolumn{10}{|l|}{ Symptoms } \\
\hline No symptoms or discomfort & 78.0 & 72.0 & 1 & & & & & & \\
\hline One or more symptoms & 73.5 & 80.3 & 0.62 & $0.37-1.05$ & 0.073 & & & & \\
\hline
\end{tabular}

related with the tumour $(7.6 \%)$. These results are similar to those in the study of Cozar et al. where the frequency of lower urinary tract symptoms was $39.5 \%$ of patients and the frequency of symptoms related to the tumour was $11.6 \%$ [16]. In our study, the median interval between first consultation and diagnosis was 89.0 days, comparable to the 72 days in the study by Hansen et al. [31] and the 101 days reported by Torring et al. [32].

We did not find any variability in diagnostic interval between hospitals regarding age, BMI, education level, first visit with a specialist, tumour stage, PSA value, or Gleason score. However, the presence of symptoms lengthened this interval possibly because some symptoms of prostate cancer can be confused with benign prostatic hyperplasia.
Previous studies in Spain that determined the therapeutic intervals in cancer patients were generally conducted in a single hospital [33-35]. The most recently published multicentre study analysed this interval for six types of cancer, including prostate cancer [19], and found the mean therapeutic interval was longer than in our study (102.5 days (SD:71.6) vs. 80.4 days (SD:60.9)). However, we defined this interval as the time between the biopsy and the first oncological treatment, whereas the investigators in the previous study defined it as the time between the first diagnostic test of any kind and first oncological treatment. In contrast with the study by Perez et al. [19], our study was prospective, it had a larger number of cases, patients were from several different autonomous regions of the country, and information 
was obtained not only from medical records but also through patient interviews.

We observed that patients with a higher PSA value and a higher Gleason score had a shorter interval between diagnosis and first treatment than patients with lower values. Pérez et al. [19] reported similar findings in patients with advanced stages of prostate cancer. An explanation for this shorter interval could be that due to their worse prognosis, these patients usually receive hormonal therapy initially or exclusively, a treatment that is easier to administer than radiotherapy, chemotherapy, or surgery [36].

Our results show a statistically significant variability between centres in relation to the therapeutic interval. The heterogeneity in intervals could be associated with the wide diversity in population characteristics, healthcare organisation and clinical policies in the different regions in Spain.

One of the main strengths of our study is that our sample of patients is a representative sample of the approximately 28.000 yearly incident prostatic cancer patients diagnosed in Spain because they were recruited from seven hospitals in five autonomous regions. In addition, the study's prospective nature guarantees consistency and accuracy of the data collected, surpassing the common shortcomings of a retrospective collection of information. The study may have limitations, however, such as information bias. Given that it is based exclusively on information obtained in a hospital setting, outpatient factors such as those related to consultation at a primary level, could not have been taken into consideration. Nevertheless, as urologic cancer care is mainly provided in the hospital setting, in our view this limitation has little practical relevance.

\section{Conclusions}

Most incident prostate cancer patients in Spain are diagnosed at an early stage of an adenocarcinoma. The period to complete the diagnostic process is approximately three months whereas the therapeutic intervals vary among centres and are shorter for patients with a worse prognosis. The presence of prostatic symptoms, PSA level, and Gleason score influence the clinical intervals differently.

\section{Appendix}

\section{EMPARO study group}

Coordinating investigator: Xavier Bonfill Cosp (Iberoamerican Cochrane Centre. Public Health and Clinical Epidemiology Service. Hospital de la Santa Creu i Sant Pau, IIB Sant Pau, Barcelona, Spain).

Project manager: $\mathrm{M}^{\mathrm{a}}$ José Martínez Zapata (Iberoamerican Cochrane Centre. IIBSant Pau, Barcelona, Spain).
Clinical research assistants: Alborada Martínez (Universidad de Valencia); Enrique Morales Olivera (Escuela Andaluza de Salud Pública, Granada, Spain); Esther Canovas, Laura Muñoz, Gemma Mas, René Acosta, Ekaterina Popova (Iberoamerican Cochrane Centre. IIBSant Pau, Barcelona, Spain); Irma Ospina (Hospital 12 de Octubre, Madrid, Spain); Ma José Velázquez (Hospital Donostia, Donostia, Spain); Tamara Ruiz Merlo (Hospital Ramón y Cajal, Madrid, Spain); Gael Combarros Herman, Judit Tirado Muñoz (IMIM, Barcelona, Spain).

Statistical analysis: Robin Vernooij (Iberoamerican Cochrane Centre. IIBSant Pau, Barcelona, Spain); Victor Abraira (Unidad de Bioestadística Clínica. Hospital Universitario Ramón y Cajal. IRYCIS, Madrid, Spain).

\section{Co-investigators:}

\section{Barcelona, Spain}

Virginia Becerra Bachito, Montserrat Ferrer Fores, Stefanie Schmidt, Olatz Garin, Yolanda Pardo (IMIM Hospital del Mar Medical Research Institute-); Albert Frances (Hospital del Mar); Carola Orrego Villagran, Rosa Suñol (Instituto U. Avedis Donabedian); Dimelza Osorio, Gemma Sancho Pardo, Ignasi Bolívar, José Pablo Maroto, $\mathrm{M}^{\mathrm{a}}$ Jesús Quintana, Martin Lorente, Cristina (Hospital de la Santa Creu i Sant Pau); Ferran Algaba, Palou Redorta, Salvador Esquena (Fundació Puigvert); Jordi Bachs (Fundació Privada Hospital de la Santa Creu i Sant Pau); Ma José Martínez Zapata (Iberoamerican Cochrane Centre. IIBSant Pau).

\section{Bilbao, Spain}

Amaia Martínez Galarza, José Ignacio Pijoán Zubizarreta, Lorea Martínez (Hospital Universitario Cruces-BioCruces Health Research Institute).

\section{Tenerife, Spain}

David Manuel Castro Diaz, Juan Manuel Ramos Goñi, Julio López Bastida (HTA Unit of the Canary Islands Health Service).

\section{Granada, Spain}

Armando Suárez Pacheco, Cesar García López, Jose Manuel Cozar Olmo (Hospital Universitario Virgen de las Nieves); Carmen Martínez, Daysy Chang Chan, $\mathrm{M}^{\mathrm{a}}$ Jose Sanchez Perez (Escuela Andaluza de Salud Pública).

\section{Madrid, Spain}

Ana Isabel Díaz Moratinos, Angel Montero Luis, Asunción Hervás, Carmen Vallejo Ocaña, Costantino Varona, Javier Burgos, Javier Zamora, Jose Alfredo Polo Rubio, Luis López-Fando Lavalle, Miguel Angel Jiménez Cidre, Muriel Garcia, Alfonso, Nieves Plana Farras, Rosa Morera López, Sonsoles Sancho Garcia, Víctor Abraira, 
Victoria Gómez Dos Santos (Hospital Ramón y Cajal); Agustín Gómez de la Cámara, Javier de la Cruz, Juan Passas Martínez, Humberto García Muñoz, Ma Ángeles Cabeza Rodríguez (Hospital 12 de Octubre).

\section{San Sebastián, Spain}

Irune Ruiz Díaz, José Ignacio Emparanza, Juan Pablo Sanz Jaka (Hospital Universitario Donostia).

\section{Valencia, Spain}

Agustín LLopis González, María Morales (Universidad de Valencia); Carlos Camps, Cristina Caballero Díaz, Emilio Marqués Vidal, Francisco Sánchez Ballester, Joaquín Ulises Juan Escudero, Jorge Pastor Peidro, José López Torrecilla, $\mathrm{M}^{\mathrm{a}}$ Macarena Ramos Campos, Miguel Martorell Cebollada (Consorcio Hospital General Universitario de Valencia).

\section{Abbreviations}

PSA: Prostate-specific antigen; SD: Standard deviation; IQR: Interquartile range; BMI: Body mass index; OR: Odds ratio; ICC: Intra-cluster correlation coefficient; MOR: Median odds ratio.

\section{Competing interests}

The authors declare that they have no competing interest.

\section{Authors' contributions}

Study concepts: XB. Study design: XB, MJM. Data acquisition: All authors. Quality control of data and algorithms: MJM, RV. Data analysis and interpretation: XB, MJM, RV. Statistical analysis: RV, VA. Manuscript preparation: XB MJM, RV. Manuscript editing: All authors. Manuscript review: All authors. All authors read and approved the final manuscript.

\section{Acknowledgements}

We would like to thank Andrea Cervera Alepuz for the English review and editing of the manuscript. Funding: Instituto Carlos III: Fondo de Investigación Sanitaria PS09/01204. Spain.

\section{Author details \\ ${ }^{1}$ CIBER de Epidemiología y Salud Pública (CIBERESP), Madrid, Spain. ${ }^{2}$ Institute of Biomedical Research (IIB Sant Pau), Iberoamerican Cochrane Centre, Barcelona, Spain. ${ }^{3}$ Universitat Autònoma de Barcelona, Barcelona, Spain. ${ }^{4}$ Public Health and Clinical Epidemiology Service, Hospital de la Santa Creu i Sant Pau, Barcelona, Spain. ${ }^{5}$ Instituto de Investigación Biosanitaria de Granada, Escuela Andaluza de Salud Pública, Granada, Spain. ${ }^{6}$ Department of Preventive Medicine, Unit of Public Health and Environmental Care, University of Valencia, Center for Public Health Research (CSISP), Valencia, Spain. ${ }^{7}$ Hospital 12 de Octubre, Madrid, Spain. ${ }^{8}$ Clinical Epidemiology Unit, Hospital Universitario Donostia, BioDonostia, San Sebastian, Spain. ${ }^{9}$ IMIM (Hospital del Mar Medical Research Institute), Health Services Research Group, Barcelona, Spain. ${ }^{10}$ Unidad de Epidemiología Clínica y Soporte Metodológico, UICEC de BioCruces-SCReN, Barakaldo, Spain. ${ }^{11}$ Health Services Research on Chronic Patients Network (REDISSEC), HTA Unit of the Canary Islands Health Service (SESCS), S/C de Tenerife, La Laguna, Spain. ${ }^{12}$ Fundació Puigvert, Barcelona, Spain. ${ }^{13}$ Department of Experimental and Health Sciences, Universidad Pompeu Fabra (UPF), Barcelona, Spain. ${ }^{14}$ Unidad de Bioestadística Clínica, Hospital Universitario Ramón y Cajal, IRYCIS, Madrid, Spain.}

Received: 18 June 2014 Accepted: 16 June 2015

Published online: 02 July 2015

\section{References}

1. Ferlay J, Soerjomataram I, Ervik M, Dikshit R, Eser S, Mathers C, et al. GLOBOCAN 2012 v1.0, Cancer incidence and mortality worldwide: IARC
CancerBase No. 11. Lyon, France: International Agency for Research on Cancer; 2013. [http://globocan.iarc.fr]. Accessed 14 Apr 2014.

2. Eastham JA, Riedel E, Scardino PT, Shike M, Fleisher M, Schatzkin A, et al Variation of serum prostate-specific antigen levels: an evaluation of year-to-year fluctuations. JAMA. 2003;289(20):2695-700.

3. Penson DF, Rossignol M, Sartor AO, Scardino PT, Abenhaim LL. Prostate cancer: epidemiology and health-related quality of life. Urology. 2008;72(6 Suppl):S3-11.

4. Stamey TA, Caldwell M, McNeal JE, Nolley R, Hemenez M, Downs J. The prostate specific antigen era in the United States is over for prostate cancer: what happened in the last 20 years? J Urol. 2004;172(4):1297-301.

5. Sánchez MJ, Payer T, De Angelis R, Larrañaga N, Capocaccia R, Martinez C. CIBERESP working group cancer incidence and mortality in Spain: estimates and projections for the period 1981-2012. Ann Oncol. 2010;21(3):30-6.

6. Larrañaga N, Galceran J, Ardanaz E, Franch P, Navarro C, Sánchez MJ, et al. Prostate cancer incidence trends in Spain before and during the prostate-specific antigen era: impact on mortality. Ann Oncol. 2010;21 Suppl 3:83-9.

7. Mickevičienè A, Vanagas G, Ulys A, Jievaltas M, Smailytè G, Padaiga Ž. Factors affecting health-related quality of life in prostate cancer patients. Scand J Urol Nephrol. 2012:46(3):180-7.

8. Litwin MS, Hays RD, Fink A, Ganz PA, Leake B, Leach GE, et al. Quality-of-life outcomes in men treated for localized prostate cancer. JAMA. 1995;273(2):129-35.

9. Mariotto AB, Yabroff KR, Shao Y, Feuer EJ, Brown ML. Projections of the cost of cancer care in the United States: 2010-2020. J Natl Cancer Inst. 2011;103:117-28.

10. Sangar VK, Ragavan N, Matanhelia SS, Watson MW, Blades RA. The economic consequences of prostate and bladder cancer in the UK. BJU Int. 2005:95(1):59-63.

11. Sant M, Allemani C, Santaquilani M, Knijn A, Marchesi F, Capocaccia R, et al. EUROCARE-4. Survival of cancer patients diagnosed in 1995-1999. Results and commentary. Eur J Cancer. 2009;45(6):931-91.

12. Salive ME, Mayfield JA, Weissman NW. Patient outcomes research teams and the agency for health care policy and research. Health Serv Res. 1990:25(5):697-708.

13. Lubeck DP, Litwin MS, Henning JM, Stier DM, Mazonson P, Fisk R, et al. The CaPSURE database: a methodology for clinical practice and research in prostate cancer. CaPSURE Research Panel. Cancer of the Prostate Strategic Urologic Research Endeavor. Urology. 1996;48(5):773-7.

14. Navarro C, Martos C, Ardanaz E, Galceran J, Izarzugaza I, Peris-Bonet R, et al Population-based cancer registries in Spain and their role in cancer control. Ann Oncol. 2010:21 suppl 3:iii3-13.

15. Calle JE, Saturno PJ, Parra P, Rodenas J, Pérez MJ, Eustaquio FS, et al. Quality of the information contained in the minimum basic data set: Results from an evaluation in eight hospitals. Eur J Epidemiol. 2000:16(11):1073-80.

16. Cózar JM, Miñana B, Gómez-Veiga F, Rodríguez-Antolín A, Villavicencio H Cantalapiedra A, et al. Registro nacional de cáncer de próstata 2010 en España [National prostate cancer registry 2010 in Spain]. Actas Urol Esp. 2013;37(1):12-9.

17. Cózar JM, Miñana B, Gómez-Veiga F, Rodríguez-Antolín A, Villavicencio H, 25 Urology Units, Asociación Española de Urología, et al. Prostate cancer incidence and newly diagnosed patient profile in Spain in 2010. BJU Int. 2012;110(11 Pt B):E701-6

18. American Joint Committee on Cancer. AJCC cancer staging manual. 6th ed. New York, NY: Springer; 2002. p. 335-40.

19. Pérez G, Porta M, Borrell C, Casamitjana M, Bonfill X, Bolibar I, et al. Interval from diagnosis to treatment onset for six major cancers in Catalonia, Spain Cancer Detect Prev. 2008:32(3):267-75.

20. Kawakami J, Hopman WM, Smith-Tryon R, Siemens DR. Measurement of surgical wait times in a universal health care system. Can Urol Assoc J. 2008;2(6):597-603.

21. Saad F, Finelli A, Dranitsaris G, Goldenberg L, Bagnell S, Gleave M, et al. Canadian surgical wait times (SWAT) initiative. Does prolonging the time to prostate cancer surgery impact long-term cancer control: a systematic review of the literature. Can J Urol. 2006;13 Suppl 3:16-24.

22. Fleshner N, Dranitsaris G, Finelli A, Tsihlias J, Bell D, Gleave M. Canadian surgical wait times (SWAT) initiative. Surgical wait times for patients with urological cancers: a survey of Canadian surgeons. Can J Urol. 2006;13 Suppl 3:3-13. 
23. Rabe-Hesketh S, Skrondal A. Multilevel and longitudinal modeling using stata. College Station, TX: Stata Press; 2008.

24. Merlo J, Chaix B, Ohlsson H, Beckman A, Johnell K, Hjerpe P, et al. A brief conceptual tutorial of multilevel analysis in social epidemiology: using measures of clustering in multilevel logistic regression to investigate contextual phenomena. J Epidemiol Community Health. 2006;60(4):290-7.

25. Gatta G, Zigon G, Buemi A, Coebergh JW, Colonna M, Contiero P, et al. Prostate cancer in Europe at the end of 1990s. Acta Oncol. 2009;48(6):867-73.

26. Andrén O, Fall K, Franzén L, Andersson S-O, Johansson J-E, Rubin MA. How well does the Gleason score predict prostate cancer death? a 20-year followup of a population based cohort in Sweden. J Urol. 2006;175:1337-40.

27. Bill-Axelson A, Garmo H, Lambe M, Bratt O, Adolfsson J, Nyberg U, et al. Suicide risk in men with prostate-specific antigen-detected early prostate cancer: a nationwide population-based cohort study from PCBaSe Sweden. Eur Urol. 2010;57:390-5.

28. Holmstrom B, Johansson M, Bergh A, Stenman U-H, Hallmans G, Stattin P. Prostate specific antigen for early detection of prostate cancer: longitudinal study. BMJ. 2009;339:b3537.

29. Smith DP, King MT, Egger S, Berry MP, Stricker PD, Cozzi P, et al. Quality of life three years after diagnosis of localised prostate cancer: population based cohort study. BMJ. 2009;339:b4817.

30. Salinas M, López-Garrigós M, Miralles F, Chinchilla V, Ortuño M, Aguado C, et al. Evaluation of PSA testing by general practitioners: regional study in the autonomic Community of Valencia. Arch Esp Urol. 2011;64(5):435-40.

31. Hansen RP, Vedsted P, Sokolowski I, Søndergaard J, Olesen F. Time intervals from first symptom to treatment of cancer: a cohort study of 2,212 newly diagnosed cancer patients. BMC Health Serv Res. 2011;11:284.

32. Tørring ML, Frydenberg M, Hansen RP, Olesen F, Vedsted P. Evidence of increasing mortality with longer diagnostic intervals for five common cancers: a cohort study in primary care. Eur J Cancer. 2013;49(9):2187-98.

33. Porta M, Gallén M, Malats N, Planas J. The influence of diagnostic delay upon cancer survival. An analysis of five tumour sites. J Epidemiol Community Health. 1991;45:225-30.

34. Maguire A, Porta M, Malats N, Gallén M, Piñol JL, Fernández E. Cancer survival and the duration of symptoms. An analysis of possible forms of the risk function. Eur J Cancer. 1994;30:785-92.

35. Fernández E, Porta M, Malats N, Belloc J, Gallén M. Symptom to diagnosis interval and survival in cancers of the digestive tract. Dig Dis Sci. 2002;47:2434-40

36. NCCN Clinical Practice Guidelines in Oncology (NCCN Guidelines ${ }^{\circledR}$ ). Prostate cancer. Version 1. 2014. NCCN.org

\section{Submit your next manuscript to BioMed Central and take full advantage of:}

- Convenient online submission

- Thorough peer review

- No space constraints or color figure charges

- Immediate publication on acceptance

- Inclusion in PubMed, CAS, Scopus and Google Scholar

- Research which is freely available for redistribution 